\title{
Protocol - A Systematic Scoping Review of the Interventions Available During Care Crises for People with Learning or Intellectual Disabilities (LD) who are Cared for by an Ageing Carer within the United Kingdom
}

\author{
Mahon $\mathrm{A}^{\mathbf{1}}$, Vseteckova $\mathrm{J}^{2 *}$, Tilley $\mathrm{E}^{\mathbf{2}}$, Pappas $\mathrm{Y}^{\mathbf{1}}$ and Randhawa $\mathrm{G}^{\mathbf{1}}$ \\ ${ }^{1}$ Institute for Health Research, University of Bedfordshire, UK \\ ${ }^{2}$ Senior Lecturer Health, Wellbeing and Social Care, Faculty of Wellbeing, The Open University, Milton Keynes, UK
}

*Corresponding author: Vseteckova J, Senior Lecturer Health, Wellbeing and Social Care, Faculty of Wellbeing, The Open

University, Milton Keynes, UK

\begin{tabular}{|c|c|}
\hline ARTICLE INFO & ABSTRACT \\
\hline Received: 幽 August 14, 2019 & Citation: Mahon A, Vseteckova J, Tilley E, Pappas Y, Randhawa G. Protocol - A Systematic \\
\hline Published: 慧 August 23, 2019 & $\begin{array}{l}\text { Scoping Review of the Interventions Available During Care Crises for People with } \\
\text { Learning or Intellectual Disabilities (LD) who are Cared for by an Ageing Carer within } \\
\text { the United Kingdom. Biomed J Sci \& Tech Res } 20(5)-2019 \text {. BJSTR. MS.ID.003512. }\end{array}$ \\
\hline
\end{tabular}

\section{Introduction}

The majority of individuals with learning disabilities within the United Kingdom (UK) are cared for by a family member, usually a parent. However, individuals with learning disabilities in the UK are now living longer than ever before [1] which means that the number of ageing family members providing care is also increasing. Furthermore, an increasing number of individuals with LD are now also outliving their parents [2]. Despite this, many adults with LD and their ageing carers do not make plans for their future [2], including periods of care crisis such as the serious illness or death of their ageing primary care provider. Yet, literature repeatedly states that one of the main concerns of ageing carers are "fears about the future". It is crucial that existing crisis interventions for individuals with LD who are cared for by an ageing carer within the UK are identified and evaluated as well as current preventative measures that assist in future care planning. Identifying these measures may reduce the occurrence of crisis periods or assist in the transition of care.

A preliminary search for existing scoping reviews and/or systematic reviews on the topic was conducted. Within the UK there are limited primary research papers and research syntheses available on this topic. To the authors knowledge no existing scoping reviews or systematic reviews on this topic currently exist. The aim of the current scoping review is to provide a greater understanding of the existing crisis interventions and preventative support available for individuals with LD who are cared for by an ageing carer within the UK. This will include evaluating the outcomes and effectiveness of available interventions, including cost-efficiency (where this information is provided). Following the PICO framework [3], this scoping review aims to synthetize existing knowledge, identify gaps in the literature and provide recommendations for future research, which may lead to improved interventions and the potential to improve future planning of care provisions for ageing individuals with LD.

\section{Methods}

A systematic scoping review was deemed the most suitable method due to the research questions proposed by the current review and the literature it intended to include. The current review aimed to synthesize all available evidence on the availability and effectiveness of the interventions available to support people with LD who are being cared for by older carers in times of crisis. This included a review of the financial implications of such crises and interventions. Crucially, systematic scoping reviews are designed to synthesize knowledge according to an exploratory research question and as such it was a suitable methodology for this review. Scoping reviews provide an overview of broad research fields and may include a wide variety of different research methodologies and literature sources. The topic of the current review is also broad and required the inclusion of a wide variety of available literature, 
specifically grey literature such as annual reports from healthcare providers in regard to the cost efficiency of identified interventions.

Lastly, as the current review included all available interventions, a systematic review was deemed inappropriate as this would only answer questions relating to specific interventions. It is important to note that while scoping reviews generally do not require quality assessments of literature included, they do share a similar methodology to systematic reviews and as such they are rigours, transparent and methodical in their approach [4-6]. As an extension of the Preferred Reporting Items for Systematic Reviews and Metaanalysis (PRISMA) statement called the Preferred Reporting Items for Systematic Reviews and Meta-Analysis: extension for Scoping Reviews (PRISMA-ScR) is currently under development [7], the current scoping review followed the methodological framework proposed by Arksey, et al. [4] as well as the amendments to this framework proposed by Levac, et al. [8], the Joanna Briggs Institute [9] and the guidelines for best practices provided by Colquhoun, et al. [10].

The Joanna Briggs Institute (JBI) methodology for Scoping Reviews, will be "congruent with the PRISMA-ScR checklist" and "will assist in standardizing future scoping reviews". It has been recommended [11] that scoping review protocols follow the relevant aspects of the Preferred Reporting Items for Systematic Reviews and Meta-analysis for Protocols (PRISMA-P) guidelines [12] thus, the PRISMA-P was used to draft this current protocol. The Arksey and O'Malley's scoping review framework consists of five consecutive stages:

a) Identifying the research question,

b) Identifying relevant studies,

c) Study selection,

d) Charting the data,

e) Collating, summarising and reporting results. Each stage is discussed in further detail below. The last optional stage, consultation, was not included in the current scoping review.

\section{Stage 1: Identifying the Research Question}

The aim of the current scoping review is to provide a greater understanding of the existing services, infrastructure, funding, policies and/or interventions (both short term/long term) within the UK to support individuals with LD who are cared for by an unpaid ageing carer in the event of a crises period, e.g. when the carer becomes acutely or chronically ill, or in the case of death of a carer. This included reviewing the literature for any cost analysis, costing models or financial implications reported in reference to crisis interventions. Following the PICO framework, this scoping review aimed to synthetize existing knowledge, identify gaps in the literature and provide recommendations for future research, which may lead to improved crisis interventions with the potential to improve the future provision of care for individuals with LD who are cared for by ageing unpaid carers.
To meet these objectives, this review asked the following questions:

a. What short term support is there for individuals (aged 40 years and upwards) with LD who are cared for by ageing carers in the United Kingdom in times of crises?

b. What long term support is there for individuals (aged 40 years and upwards) with LD who are cared for by ageing carers in the United Kingdom in times of crises?

c. Are there any costing model, cost analysis, or financial implications reported in reference to crisis interventions?

d. Are there any existing early interventions (preventive measures to such crisis) for individuals (aged 40 years and upwards) with LD who are cared for by ageing carers in the United Kingdom?

e. Is there a difference in outcomes, either financially, psychologically, physiologically or socially, when crisis interventions are available and implemented compared to instances in which individuals with LD are not offered any crisis support or where early interventions have been introduced to prevent such crises?

\section{Stage 2: Identifying Relevant Studies}

Scoping reviews provide an overview of broad research fields [6] as such the current review used a wide and diverse search to source all relevant studies. This included a search of electronic databases, reference lists (ancestor searching), website organisations and conference proceedings. Articles and evaluation reports related to the topic of ageing carers of individuals with learning disabilities were identified through an initial exploratory online search using the electronic databases MEDLINE (PubMed) and CINAHL. The text words in the title and abstract of relevant retrieved papers were then analysed as well as the index terms used to describe the articles. All identified keywords and index terms were used to develop a rigorous search strategy that was undertaken across all included databases. The reference list of identified reports and articles was also searched for additional studies. The reviewers' contacted authors of primary studies or reviews for further information, where relevant. The search was limited to literature written in English. No date restrictions were applied. The search strategy and details of the search dates can be found in Appendix 1.

\section{Inclusion Criteria}

\section{A. Types of Participants}

Eligible participants included:

a) Individuals with learning disabilities (LD), aged 40+ living within the United Kingdom whose main carer is an unpaid ageing carers (aged 65 years and over).

b) Carers must not be caring for the individual with learning disabilities as part of their professional vocation i.e. they will be unpaid parents, family members or friends. 
c) Providers and/or stakeholders involved in the formulation, commissioning or provision of short- and longterm interventions for the care for individuals with learning disabilities (LD), aged 40+ living within the United Kingdom whose main carer is an unpaid ageing carers in periods of crisis.

d) Providers and/or stakeholders involved in the formulation, commissioning or provision of early interventions for the care for individuals with learning disabilities (LD), aged $40+$ living within the United Kingdom whose main carer is an unpaid ageing carers in the event of a future crisis.

\section{B. Concept}

\section{a. Types of Outcome Measures}

To be included in the review, records had to report at least one of the following outcome measures:

\section{Primary Outcome}

The outcomes were expected to be diverse and context-specific. The primary outcomes of interest where

a) The identification of existing services, infrastructure, funding, policies and/or interventions (both short term/long term) within the UK to support individuals with LD who are cared for by an unpaid ageing carer in the event of a crisis period.

b) To review the effectiveness of identified services, infrastructure, funding, policies and/or interventions (both short term/long term) within the UK to support individuals with LD who are cared for by an unpaid ageing carer in the event of a crisis. This may include but is not limited to: any quantitative feedback and opinions provided by service users before, during or after a crisis, their carers and/or professionals, any reference to any impacts interventions may have had on the mental wellbeing of patients e.g. reported management, reduction or increase in anxiety, depression and social engagement of service users, any reference to any changes in physical health, any reference to financial implications including any financial implication to the service user, their carer or family or the commissioning body/care provider.

\section{Secondary Outcomes}

The secondary outcomes of interest are the availability and effectiveness of preventative interventions that exist in the event of a future crisis as well as any impact of instances in which no intervention is available for individuals with LD who are cared for by ageing carers in periods of crises. The outcomes are expected to be diverse and context-specific. They will include:

a) The identification of existing preventative services, infrastructure, funding, policies and/or interventions (both short term/long term) within the UK to support individuals with LD who are cared for by an unpaid ageing carer in the event of a future crisis period. b) To review the effectiveness of identified preventative services, infrastructure, funding, policies and/or interventions (both short term/long term) within the UK to support individuals with LD who are cared for by an unpaid ageing carer in the event of a future crisis. This may include, quantitative feedback and opinions provided by service users, their carers and/or professionals before, during or after a crisis, any reference to any impacts interventions may have had on the mental wellbeing of patients e.g. reported management, reduction or increase in anxiety, depression and social engagement of service users, any reference to any changes in physical health, any reference to financial implications including any financial implication to the service user, their carer or family or the commissioning body/care provider.

c) To review any instances in which crisis intervention is not available and the impact of this on individuals with LD and their ageing carers. This may include, quantitative feedback and opinions provided by service users, their carers and/or professionals before, during or after a crisis, any reference to any impacts interventions may have had on the mental wellbeing of patients e.g. reported management, reduction or increase in anxiety, depression and social engagement of service users, any reference to any changes in physical health, any reference to financial implications including any financial implication to the service user, their carer or family or the commissioning body/care provider.

d) To compare the previously identified financial and psychological outcomes of crisis interventions, preventative crisis interventions and no interventions for individuals with LD within the UK who are cared for by an ageing carer.

\section{Types of Interventions}

The aim of this systematic scoping review is to synthesize knowledge regarding all available information related to the exploratory research questions, as such all interventions will be included.

\section{Context/Setting}

Both carers and the individuals they care for must live within the United Kingdom. If the carer or the individuals they are caring did not live in the United Kingdom they were excluded. Interventions that were implemented in the UK were included in the review. Those interventions that were implemented outside of the UK were excluded.

\section{b. Types of Studies}

The purpose of the current scoping review was to synthesize all relevant available knowledge. To provide a comprehensive overview of this research topic all existing literature was included, e.g. primary research studies, systematic reviews, meta-analyses, letters, guidelines, websites etc.

c. Electronic Searches

The following electronic databases were searched: 
a) CINAHL

b) British Nursing Index

c) Web of Science

d) Cochrane library

e) Medline

f) PsychInfo

g) SocIndex

h) University of York Centre for Reviews and Dissemination (DARE, NHS EED, HTA)

i) JBI Database of Systematic Reviews and Implementation Reports,

j) PubMed

k) EPPI

l) Epistemonikos,

To identify relevant evaluations in UK settings, the websites of the following organisations were searched:
a) The Kings Fund
b) The Health foundation
c) NESTA
d) NICE
e) Nuffield Trust
f) Department of Health

Additionally, grey literature was searched in OpenGrey, Google, and Google Scholar. The search terms "ageing carers of learning disabilities crisis intervention" and "older carers of learning disability crisis prevention" were used to identify grey literature because they were identified as the most relevant terms in the exploratory and database searches.

\section{d. Searching Other Resources}

In addition, reference lists of all relevant studies, reviews, and reports were searched.

Via contacting the British Library and other University libraries, the researcher obtained a full text PDF or an abstract containing sufficient details to determine eligibility of all potentially relevant studies.

\section{Stage 3: Study Selection}

\section{Study Screening and Selection}

Study selection (both at title/abstract screening and full text screening) was performed by three reviewers, independently. Any disagreements were solved by consensus or by the decision of a fourth reviewer were necessary. After eliminating the duplicates (studies that were identified more than once by the search engines), an initial screening of titles, abstracts, and summaries (if applicable) was undertaken to exclude records that clearly did not meet the inclusion criteria. Each record was classified as 'include' or 'exclude' to identify relevant and exclude irrelevant literature.

The researchers were inclusive at this stage and, if uncertain about the relevance of a publication or report, it was left in. The full text was obtained for all the records that potentially met the inclusion criteria (based on the title and abstract/summary only). In a second step, all the full text papers were screened against the inclusion criteria, using a standardised tool. Studies that did not meet the inclusion criteria were listed with the reasons for exclusion. Multiple publications and reports on the same interventions were linked together and compared for completeness. The record containing the most complete data on any single intervention was identified as the primary article in the review, which was usually the original study or most recent evaluation report. An adapted PRISMA (Preferred Reporting Items for Systematic Reviews and Meta-analyses) flow-chart of study selection was included in the review [12].

\section{Stage 4: Charting the Data}

\section{Data Extraction and Management}

Data for analysis were extracted from the included studies and managed in an Excel spread sheet. A data extraction sheet was developed which was tailored to the requirements of the review. The data extraction sheet was tested on three included papers and, where necessary, it was revised to ensure it can be reliably interpreted and could capture all relevant data from different study designs. Extracted data included authors, year of study/ report, aim/purpose, type of paper (e.g. journal article, annual evaluation report, etc.), geographical area, study population (e.g., age of participants and learning disability diagnosed), sample size, study design, description of crisis, description of the service, infrastructure, funding, policy and/or intervention (including whether it was preventative, in response to a crisis or if no intervention was available), duration of interventions, analysis used, outcomes reported by service user/carer/healthcare professional/ family unit, any clinical outcomes reported (e.g. mental wellbeing of service user such as anxiety), any financial outcomes reported (costing models; financial implications reported by family/service user/care providers), key findings that relate to the scoping review questions.

\section{Stage 5: Collating, Summarising and Reporting the Results}

\section{Presentation of the Results (Data synthesis)}

Findings from included studies were synthesised narratively. The 'Guidance on the Conduct of Narrative Synthesis in Systematic Reviews' was used to advise the narrative synthesis [13]. First, a preliminary synthesis was conducted to develop an initial description of the findings of included records and to organise them 
so that patterns across records could be identified. In a second step, thematic analysis was used to analyse the findings. The following five steps of thematic analysis were followed adopting a recursive process [14]:
a) Familiarisation with the extracted data
b) Generation of initial codes
c) Searching for themes
d) Reviewing themes
e) Defining and naming themes

Depending on the findings available the reviewers will aim to provide a flow chart mapping the interventions available for ageing carers of individuals with learning disabilities, for the needs that were identified.

\section{References}

1. Walker C, Ward C (2013) Growing older together: ageing and people with learning disabilities and their family carers. Tizard Learning Disability Review 18(3): 112-119.

2. Bibby R (2013) I hope he goes first: Exploring determinants of engagement in future planning for adults with a learning disability living with ageing parents. What are the issues? British Journal of Learning Disabilities 41(2): 94-105.

3. Richardson WS, Wilson MC, Nishikawa J, Hayward RS (1995) The wellbuilt clinical question: a key to evidence-based decisions. ACP journal club 123(3): A12.

ISSN: 2574-1241

DOI: 10.26717/BJSTR.2019.20.003512

Vseteckova J. Biomed J Sci \& Tech Res

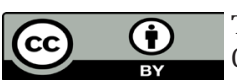

This work is licensed under Creative Commons Attribution 4.0 License

Submission Link: https://biomedres.us/submit-manuscript.php
4. Arksey H, O Malley L (2005) Scoping studies: towards a methodological framework. Int J Soc Res Methodol 8(1): 19-32.

5. Halas G, Schultz AS, Rothney J, Goertzen L, Wener P, et al. (2015) A scoping review protocol to map the research foci trends in tobacco control over the last decade. BMJ open 5(1): e006643.

6. Pham MT, Rajić A, Greig JD, Sargeant JM, Papadopoulos A, et al. (2014) A scoping review of scoping reviews: advancing the approach and enhancing the consistency. Research synthesis methods 5(4): 371-385.

7. Tricco A, Strauss S, Moher D (2015) Preferred Reporting Items for Systematic Reviews and Meta-Analysis: extension for Scoping Reviews (PRISMA-ScR). Equator Network.

8. Levac D, Colquhoun H, O Brien KK (2010) Scoping studies: advancing the methodology. Implement Sci 20(5): 69.

9. (2015) Joanna Briggs Institute. The Joanna Briggs Institute reviewer's manual 2015: methodology for JBI scoping reviews. Adelaide, Australia: University of Adelaide.

10. Colquhoun H (2016) Current Best Practices for the Conduct of Scoping Reviews. EQUATOR Netw 2016

11. Shamseer L, Moher D, Clarke M, Ghersi D, Liberati A, et al. (2015) Preferred reporting items for systematic review and meta-analysis protocols (PRISMA-P) 2015: elaboration and explanation. Bmj 349: g7647.

12. Moher D, Liberati A, TetzlaffJ, Altman DG, Prisma Group (2009) Preferred reporting items for systematic reviews and meta-analyses: the PRISMA statement. PLoS medicine 6(7): e1000097.

13. Popay J, Roberts H, Sowden A, Petticrew M, Arai L, et al. (2006) Guidance on the conduct of narrative synthesis in systematic reviews. A product from the ESRC methods programme Version 1: b92.

14. Braun V, Clarke V (2006) Using thematic analysis in psychology. Qualitative research in psychology 3(2): 77-101.

$\begin{array}{ll}\text { BIOMEDICAL } & \text { Assets of Publishing with us } \\ \text { RESEARCHES } & \text { - Global archiving of articles } \\ & \text { - Immediate, unrestricted online access } \\ & \text { - Rigorous Peer Review Process } \\ \end{array}$

